\title{
Topology Detection for Output-Coupling Weighted Complex Dynamical Networks with Coupling and Transmission Delays
}

\author{
Xinwei Wang, Guo-Ping Jiang, Chunxia Fan, and Xu Wu \\ College of Automation, Nanjing University of Posts and Telecommunications, Nanjing 210003, China \\ Correspondence should be addressed to Guo-Ping Jiang; jianggp@njupt.edu.cn
}

Received 6 November 2016; Revised 1 April 2017; Accepted 18 June 2017; Published 31 July 2017

Academic Editor: Alessandro Lo Schiavo

Copyright (c) 2017 Xinwei Wang et al. This is an open access article distributed under the Creative Commons Attribution License, which permits unrestricted use, distribution, and reproduction in any medium, provided the original work is properly cited.

\begin{abstract}
Topology detection for output-coupling weighted complex dynamical networks with two types of time delays is investigated in this paper. Different from existing literatures, coupling delay and transmission delay are simultaneously taken into account in the output-coupling network. Based on the idea of the state observer, we build the drive-response system and apply LaSalle's invariance principle to the error dynamical system of the drive-response system. Several convergent criteria are deduced in the form of algebraic inequalities. Some numerical simulations for the complex dynamical network, with node dynamics being chaotic, are given to verify the effectiveness of the proposed scheme.
\end{abstract}

\section{Introduction}

Complex networks have various existence $[1,2]$ ranging from the World-Wide Web to neural networks, from cell phone webs to social networks [3], from food webs to metabolic networks [4], and so on [5, 6]. Since the small-world [7] and scale-free [8] network models were proposed, researches on the complex network have entered a new stage. Abundant research achievements have been concentrated on many perspectives like exploration on the dynamics $[9,10]$ and complexity [11] of the complex network, synchronization criteria $[12,13]$ and control strategies [14-16] in complex networks, estimation of uncertain state variables in continuous [17-19] or discrete [20] time domain under the fixed topology, and so on. In practical circumstances, the topological structure of a complex network is barely known exactly due to the weak cognition to the network complexity. If a serious malfunction occurs in a large-scale network, it is primary to quickly identify the fault location before trouble removal. Therefore, topology detection of complex dynamical networks [21-29] has become a significant topic for further studies. It is functionally powerful to employ the adaptive feedback control strategy to the structure detection of complex networks [21, 22, 24-28]. The target topology matrix which Zhou and Lu [21] considered was not needed to be symmetric, irreducible, or diffusive. If nodes in their network had different dynamics, the detection could still be accomplished. Additionally, stochastic perturbations [22] were considered in the structure identification of complex networks. In [23], based on the generalized outer synchronization, the topological structure of the complex network was recovered by constructing the auxiliary response network which had simpler node dynamics.

Recently, the coupling delay has been taken into account for the more realistic topology detection [24-27] since it is ubiquitous in the interactions of network nodes. Sometimes it leads to additional complex dynamics [24]. In [24], the topology of the complex network with coupling delays was successfully identified by an adaptive feedback controller. Their approach was applied to complex networks with not only identical nodes but also different nodes. The inner coupling matrix in [24] was chosen as a linear one. The topology detection problem for weighted timevarying dynamical networks with nonlinear inner coupling was addressed in [25]. Besides the topology detection, the estimation for uncertain system parameters [26] was conducted in general complex delayed dynamical networks [27]. It provides positive possibility for future applications. However, in [21-27], information signals were assumed to be transmitted as instant communication between the drive and response systems, which is not practical enough. Due to long communication distance and unexpected transmission 
congestions, it definitely takes finite time for exchanging messages through the channel and the time delay should not be ignored. In addition, the inevitable transmission delay in reality is always hard to determine. Based on the statecoupling network model, the unknown network structure was obtained by achieving the lag synchronization between drive and response systems [28]. In their scheme, all states of nodes were transmitted for the synchronization of full statecoupling complex networks.

The output-coupling network [14] whose every two nodes connect to each other via just a scalar signal saves interior communication resource, which has received more attention recently. The transmission signals between the drive and response output-coupling networks are also scalar outputs instead of state vectors. It further reduces the capacity of communication channels and becomes more available for real engineering. Fan et al. [29] detected the structures for statecoupling and output-coupling complex networks, respectively. Numerical examples verified their scheme effective applying to different kinds of networks without any time delay.

Motivated by the above discussions, study on the topology detection of output-coupling complex networks with both coupling and transmission delays is increasingly closer to the reality and has very important research significance. In this paper, based on the output-coupling network model, we investigate the topology detection problem considering coupling and transmission delays at the same time. The transmission delay does not need to be known exactly, which makes our scheme applicable for a more general case. The target network serves as the drive system and a response system is built accordingly whose node dynamics is the same as the drive system. The topology of the target network can be directed and weighted. Applying LaSalle's invariance principle $[24,25,30]$, the error dynamical system is stabilized to approaching zero asymptotically while the structure detection is completed and topology changes are tracked in time.

The rest parts of this paper are organized as follows. The output-coupling complex network model with the coupling delay is described in Section 2. Topology detection of a general output-coupling complex network with coupling and transmission delay is discussed in detail and some convergent criteria are given in the form of algebraic inequalities in Section 3. In Section 4, several convincing simulation results are shown to confirm the accuracy and correctness of our proposed strategy. Some conclusions are provided in Section 5 .

\section{Preliminaries}

Consider the output-coupling complex dynamical network with coupling delays composed of $N$ identical nodes, which is described by

$$
\begin{aligned}
& \dot{x}_{i}(t)=f\left(x_{i}(t)\right)+\sum_{j=1}^{N} c_{i j}(t) L y_{j}\left(t-\tau_{1}\right), \\
& y_{i}(t)=H x_{i}(t), \quad \\
& \quad i=1,2, \ldots, N, N
\end{aligned}
$$

where $x_{i}(t)=\left[x_{i 1}(t), x_{i 2}(t), \ldots, x_{i n}(t)\right]^{T} \in R^{n}$ is the state vector of the $i$ th node and $y_{i} \in R$ is the output scalar of that. $f: R \times R^{n} \rightarrow R^{n}$ is a smooth nonlinear vector field. $H \in R^{1 \times n}$ is the output matrix of each node. In this way, $y_{i}$ is a linear combination of the $i$ th node's state components, and $L \in R^{n \times 1}$ is the inner coupling matrix. $\tau_{1}$ is the inner coupling delay in the complex network. For simplicity, we assume that coupling delays of all nodes are the same constant value in this paper. $C(t)=\left(c_{i j}(t)\right)_{N \times N} \in R^{N \times N}$ is the uncertain configuration matrix which represents the coupling strength and topological structure of the complex network. If there exists a connection from node $i$ to node $j(i \neq j)$, then $c_{i j}(t) \neq$ 0 ; otherwise $c_{i j}(t)=0$. Assume that the diagonal elements $\left\{c_{i i}(t) \mid i=1,2, \ldots, N\right\}$ of $C(t)$ satisfy $c_{i i}(t)=-\sum_{j=1, j \neq i}^{N} c_{i j}(t)$. Here, the configuration matrix $C(t)$ is not required to be irreducible, symmetric, or diffusive.

Remark 1. $C(t)$ could switch anytime, which is used to describe the unexpected changes or manual operations on the network topological structure. It is assumed that the switching of $C(t)$ is relatively slow. Otherwise, the identification will be difficult to achieve in time, because the topology estimators need some time to react to the swift change of the topology.

Two assumptions and one lemma are introduced in the following.

Assumption 2 (A2). Suppose that there exists a nonnegative constant $\alpha$ satisfying

$$
\|f(x(t))-f(y(t))\| \leq \alpha\|x(t)-y(t)\|,
$$

which holds for any vectors $x(t), y(t) \in R^{n}$, where $\|\cdot\|$ represents the Euclidean normal.

Assumption 3 (A3). Suppose that, for any given $i, j \in\{1,2$, $\ldots, N\},\left\{L y_{j}\left(t-\tau_{1}\right)\right\}_{j=1}^{N}$ is linearly independent of the orbit $\left\{y_{i}\left(t-\tau_{1}\right)\right\}_{i=1}^{N}$ of the synchronization manifold [31,32].

Lemma 4 (L4). For any vectors $x(t), y(t) \in R^{n}$, the inequality $2 x^{T} y \leq x^{T} x+y^{T} y$ holds for any $t$.

Remark 5. (A2) is easy to satisfy for a large class of nonlinear systems, which means the nonlinear function $f(x)$ is Lipschitz-continuous. It characterizes the property of the node dynamics. In [31-33], all the coupling terms must be linearly independent of the synchronization manifold between the drive and response systems. (A3) is a necessary condition for the purpose of detecting the network topology. Also, the coupling delay $\tau_{1}$ helps to prevent the synchronization of the drive and response systems before topology detection is completed. The transmission delay $\tau_{2}$ will be explained in the next section because it is generated by transmitting output signals between the drive and response systems.

\section{Topology Detection for the Complex Dynamical Network with the Transmission Delay}

In order to identify unknown coupling strengths, namely, the complex network's topology, we establish a drive-response 
system. We consider the network model (1) as the drive system, and the response system could be constructed as

$$
\begin{aligned}
\dot{\hat{x}}_{i}(t)= & f\left(\widehat{x}_{i}(t)\right)+\sum_{j=1}^{N} \widehat{c}_{i j}(t) L \widehat{y}_{j}\left(t-\tau_{1}\right) \\
& -k_{i}\left(\widehat{y}_{i}(t)-y_{i}\left(t-\tau_{2}\right)\right) \\
\widehat{y}_{i}(t)= & H \widehat{x}_{i}(t)
\end{aligned}
$$

where $i=1,2, \ldots, N, \widehat{x}_{i}(t)=\left[\widehat{x}_{i 1}(t), \widehat{x}_{i 2}(t), \ldots, \widehat{x}_{i n}(t)\right]^{T} \in R^{n}$ is the state vector of the $i$ th node, $\hat{y}_{i} \in R$ is the output scalar of that in the response system, $k_{i} \in\left[k_{i 1}, k_{i 2}, \ldots, k_{i n}\right]^{T}$ is the $i$ th node's observer gain which needs to be obtained, and $\tau_{2}$ is the transmission time delay which is the time spent in the communication channel. Here, the transmission delay $\tau_{2}$ is assumed to be an arbitrary constant value.

Let $e_{i}(t)=\widehat{x}_{i}(t)-x_{i}\left(t-\tau_{2}\right), e_{y i}(t)=\widehat{y}_{i}(t)-y_{i}\left(t-\tau_{2}\right)$, and $\widetilde{c}_{i j}(t)=\widehat{c}_{i j}(t)-c_{i j}\left(t-\tau_{2}\right)$; then the error dynamical system (4) could be derived from the drive system (1) and response system (3):

$$
\begin{aligned}
\dot{e}_{i}(t)= & \dot{\hat{x}}_{i}(t)-\dot{x}_{i}\left(t-\tau_{2}\right) \\
= & f\left(\widehat{x}_{i}(t)\right)-f\left(x_{i}\left(t-\tau_{2}\right)\right) \\
& +\sum_{j=1}^{N} \widehat{c}_{i j}(t) L \widehat{y}_{j}\left(t-\tau_{1}\right) \\
& -\sum_{j=1}^{N} c_{i j}\left(t-\tau_{2}\right) L y_{j}\left(t-\tau_{1}-\tau_{2}\right) \\
& -k_{i}\left(\widehat{y}_{i}(t)-y_{i}\left(t-\tau_{2}\right)\right) \\
= & f\left(\widehat{x}_{i}(t)\right)-f\left(x_{i}\left(t-\tau_{2}\right)\right) \\
& +\sum_{j=1}^{N} \widehat{c}_{i j}(t) L \widehat{y}_{j}\left(t-\tau_{1}\right) \\
& +\sum_{j=1}^{N} c_{i j}\left(t-\tau_{2}\right) L e_{y j}\left(t-\tau_{1}\right)-k_{i} e_{y i}(t) . \\
& -\sum_{j=1}^{N} c_{i j}\left(t-\tau_{2}\right) L \widehat{y}_{j}\left(t-\tau_{1}\right) \\
& +\sum_{j=1}^{N} \widetilde{c}_{i j}(t) L \widehat{y}_{j}\left(t-\tau_{1}\right) \\
& +\sum_{j=1}^{N} c_{i j}\left(t-\tau_{2}\right) L \widehat{y}_{j}\left(t-\tau_{1}\right) \\
& -\sum_{j=1}^{N} c_{i j}\left(t-\tau_{2}\right) L y_{j}\left(t-\tau_{1}-\tau_{2}\right)-k_{i} e_{y i}(t) \\
& f\left(x_{i}\left(t-\tau_{2}\right)\right) \\
& \\
&
\end{aligned}
$$

From (4), the output error dynamical system is obtained:

$$
\begin{aligned}
\dot{e}_{y i}(t)= & H \dot{e}_{i}(t) \\
= & H\left(f\left(\widehat{x}_{i}(t)\right)-f\left(x_{i}\left(t-\tau_{2}\right)\right)\right) \\
& +H\left(\sum_{j=1}^{N} \widetilde{c}_{i j}(t) L \widehat{y}_{j}\left(t-\tau_{1}\right)\right) \\
& +H\left(\sum_{j=1}^{N} c_{i j}\left(t-\tau_{2}\right) L e_{y j}\left(t-\tau_{1}\right)\right) \\
& -H k_{i} e_{y i}(t) .
\end{aligned}
$$

Theorem 6. Suppose that (A2) and (A3) hold. Use the following control law:

$$
\dot{\hat{\mathcal{c}}}_{i j}(t)=-e_{y i}^{T}(t) H L \widehat{y}_{j}\left(t-\tau_{1}\right) .
$$

If there exist matrices $k_{i}(i=1,2, \ldots, N)$ satisfying the inequality

$$
\begin{aligned}
& \left(\alpha+\lambda_{\max }\left(\frac{1}{2} P\left(t-\tau_{2}\right) P^{T}\left(t-\tau_{2}\right)\right)+\frac{1}{2}\right. \\
& \left.-\min \left(H k_{i}\right)\right)<0,
\end{aligned}
$$

where $\lambda_{\max }\left((1 / 2) P\left(t-\tau_{2}\right) P^{T}\left(t-\tau_{2}\right)\right)$ denotes the maximum eigenvalue of the matrix $\left((1 / 2) P\left(t-\tau_{2}\right) P^{T}\left(t-\tau_{2}\right)\right)$ and $\min \left(H k_{i}\right)$ denotes the minimum value among $\left\{H k_{i} \mid i=\right.$ $1,2, \ldots, N\}, P\left(t-\tau_{2}\right)=C\left(t-\tau_{2}\right) \otimes H L$. As a result, the drive system (1) and the response system (3) will achieve synchronization asymptotically and the original topology matrix $C(t)$ will be detected by $\widehat{C}(t)$ eventually; that is,

$$
\begin{aligned}
\lim _{t \rightarrow \infty}\left\|e_{y i}(t)\right\| & =0, \\
\lim _{t \rightarrow \infty}\|\widehat{C}(t)-C(t)\| & =0,
\end{aligned}
$$

$$
i, j=1,2, \ldots, N \text {. }
$$

Proof. Consider the scalar function $V$ as

$$
\begin{aligned}
V= & \frac{1}{2} \sum_{i=1}^{N} e_{y i}{ }^{T}(t) e_{y i}(t)+\frac{1}{2} \sum_{i=1}^{N} \sum_{j=1}^{N} \widetilde{c}_{i j}^{2}(t) \\
& +\frac{1}{2} \int_{t-\tau_{1}}^{t} \sum_{i=1}^{N} e_{y i}^{T}(\theta) e_{y i}(\theta) d \theta .
\end{aligned}
$$

Calculate the derivative of the scalar function (9) with the control law (6) and we get

$$
\begin{aligned}
\dot{V}= & \sum_{i=1}^{N} e_{y i}^{T}(t) \dot{e}_{y i}(t)+\sum_{i=1}^{N} \sum_{j=1}^{N} \widetilde{c}_{i j}(t) \dot{\hat{c}}_{i j}(t) \\
& +\frac{1}{2} \sum_{i=1}^{N} e_{y i}^{T}(t) e_{y i}(t)-\frac{1}{2} \sum_{i=1}^{N} e_{y i}^{T}\left(t-\tau_{1}\right) e_{y i}\left(t-\tau_{1}\right)
\end{aligned}
$$




$$
\begin{aligned}
= & \sum_{i=1}^{N} e_{y i}^{T}(t) H\left(f\left(\widehat{x}_{i}(t)\right)-f\left(x_{i}\left(t-\tau_{2}\right)\right)\right) \\
& +\sum_{i=1}^{N} \sum_{j=1}^{N} \widetilde{c}_{i j}(t) e_{y i}^{T}(t) H L \widehat{y}_{j}\left(t-\tau_{1}\right) \\
& +\sum_{i=1}^{N} \sum_{j=1}^{N} c_{i j}\left(t-\tau_{2}\right) e_{y i}^{T}(t) H L e_{y j}\left(t-\tau_{1}\right) \\
& -\sum_{i=1}^{N} e_{y i}^{T}(t) H k_{i} e_{y i}(t)+\sum_{i=1}^{N} \sum_{j=1}^{N} \widetilde{c}_{i j}(t) \dot{\hat{c}}_{i j}(t) \\
& +\frac{1}{2} \sum_{i=1}^{N} e_{y i}^{T}(t) e_{y i}(t)-\frac{1}{2} \sum_{i=1}^{N} e_{y i}^{T}\left(t-\tau_{1}\right) e_{y i}\left(t-\tau_{1}\right) \\
= & \sum_{i=1}^{N} e_{y i}^{T}(t) H\left(f\left(\widehat{x}_{i}(t)\right)-f\left(x_{i}\left(t-\tau_{2}\right)\right)\right) \\
& +\sum_{i=1}^{N} \sum_{i=1}^{N} e_{y i}^{T}\left(t-\tau_{1}\right) e_{y i}\left(t-\tau_{1}\right) . \\
& -\sum_{i=1}^{N} e_{j=1}^{T}(t) H k_{i j} e_{y i}(t)+\frac{1}{2} \sum_{i=1}^{N} e_{y i}^{T}(t) e_{y i}(t) \\
& \left.\tau_{2}\right) e_{y i}^{T}(t) H L e_{y j}\left(t-\tau_{1}\right)
\end{aligned}
$$

According to (A2), (A3), and (L4), we have

$$
\begin{aligned}
\dot{V} \leq & \sum_{i=1}^{N} e_{y i}^{T}(t) \alpha e_{y i}(t) \\
& +\sum_{i=1}^{N} \sum_{j=1}^{N} c_{i j}\left(t-\tau_{2}\right) e_{y i}^{T}(t) H L e_{y j}\left(t-\tau_{1}\right) \\
& -\sum_{i=1}^{N} e_{y i}^{T}(t) H k_{i} e_{y i}(t)+\frac{1}{2} \sum_{i=1}^{N} e_{y i}^{T}(t) e_{y i}(t)-\frac{1}{2} \\
& \cdot \sum_{i=1}^{N} e_{y i}^{T}\left(t-\tau_{1}\right) e_{y i}\left(t-\tau_{1}\right) \leq \alpha e_{y}^{T}(t) e_{y}(t)+e_{y}^{T}(t) \\
& \cdot P\left(t-\tau_{2}\right) e_{y}\left(t-\tau_{1}\right)-\min \left(H k_{i}\right) e_{y}^{T}(t) e_{y}(t)+\frac{1}{2} \\
& \cdot e_{y}^{T}(t) e_{y}(t)-\frac{1}{2} e_{y}^{T}\left(t-\tau_{1}\right) e_{y}\left(t-\tau_{1}\right) \leq \alpha e_{y}^{T}(t) \\
& \cdot e_{y}(t)+\frac{1}{2} e_{y}^{T}(t) P\left(t-\tau_{2}\right) P^{T}\left(t-\tau_{2}\right) e_{y}(t)+\frac{1}{2} \\
& \cdot e_{y}^{T}\left(t-\tau_{1}\right) e_{y}\left(t-\tau_{1}\right)-\min \left(H k_{i}\right) e_{y}^{T}(t) e_{y}(t) \\
& +\frac{1}{2} e_{y}^{T}(t) e_{y}(t)-\frac{1}{2} e_{y}^{T}\left(t-\tau_{1}\right) e_{y}\left(t-\tau_{1}\right) \leq(\alpha \\
&
\end{aligned}
$$

$$
\begin{aligned}
& +\lambda_{\max }\left(\frac{1}{2} P\left(t-\tau_{2}\right) P^{T}\left(t-\tau_{2}\right)\right)+\frac{1}{2} \\
& \left.-\min \left(H k_{i}\right)\right) e_{y}^{T}(t) e_{y}(t),
\end{aligned}
$$

where $e_{y}(t)=\left[e_{y 1}^{T}(t), e_{y 2}^{T}(t), \ldots, e_{y N}^{T}(t)\right]^{T} \in R^{N}$, and hence we get

$$
\begin{aligned}
\dot{V} & \leq\left(\alpha+\lambda_{\max }\left(\frac{1}{2} P\left(t-\tau_{2}\right) P^{T}\left(t-\tau_{2}\right)\right)+\frac{1}{2}\right. \\
& \left.-\min \left(H k_{i}\right)\right) e_{y}^{T}(t) e_{y}(t) .
\end{aligned}
$$

Taking $\left(\alpha+\lambda_{\max }\left((1 / 2) P\left(t-\tau_{2}\right) P^{T}\left(t-\tau_{2}\right)\right)+1 / 2-\right.$ $\left.\min \left(H k_{i}\right)\right)<0$ from (7), we have $\dot{V} \leq 0$. Evidently, $\dot{V}=0$ if and only if $e_{y}(t)=0$; then $E=\{\dot{V}=0\}=\left\{e_{y}(t)=0\right\}$. Along with the output error dynamical system (5), if $\widehat{C}(t)-$ $C(t) \neq 0$, then $\dot{e}_{y i}(t) \neq 0, e_{y i}(t)$ would not be fixed at the zero point. That means the largest invariant set $M$ contained in $E$ is $M=\left\{e_{y}(t)=0, \widehat{C}(t)-C(t)=0\right\}$. According to LaSalle's invariance principle [29], with arbitrary initial values of the output error dynamical system (5), the trajectories converge asymptotically to the set $M$, which ensures us the detection quality of the complex network's topology. The proof is completed.

\section{Numerical Simulations}

In this section, a representative example is shown to verify the effectiveness of the topology detection scheme proposed in Section 3. Here, the node dynamics is characterized by the chaotic system. The Lorenz system is one of the most typical chaotic systems, which could be described by

$$
\begin{aligned}
& \dot{x}_{i 1}(t)=a\left(x_{i 2}(t)-x_{i 1}(t)\right), \\
& \dot{x}_{i 2}(t)=c x_{i 1}(t)-x_{i 2}(t)-x_{i 1}(t) x_{i 3}(t), \\
& \dot{x}_{i 3}(t)=x_{i 1}(t) x_{i 2}(t)-b x_{i 3}(t),
\end{aligned}
$$

when $a=10, b=8 / 3$, and $c=28$, and the Lorenz system will show the chaotic characteristics. For any two state vectors $x_{i}$ and $x_{j}$ of the Lorenz system, there exists a constant $\theta$ satisfying $\left\|x_{i k}\right\| \leq \theta$ and $\left\|x_{j k}\right\| \leq \theta$ since chaotic attractor is bounded in a certain region; thus we obtain

$$
\begin{aligned}
& \left\|f\left(x_{i}\right)-f\left(x_{j}\right)\right\| \\
& \quad=\sqrt{\left(x_{j 1} x_{j 3}-x_{i 1} x_{i 3}\right)^{2}+\left(x_{i 1} x_{i 2}-x_{j 1} x_{j 2}\right)^{2}} \\
& \quad \leq 2 \theta\left\|x_{i}-x_{j}\right\|=\alpha\left\|x_{i}-x_{j}\right\| .
\end{aligned}
$$

Obviously, (A2) can be easily satisfied in the Lorenz system. 


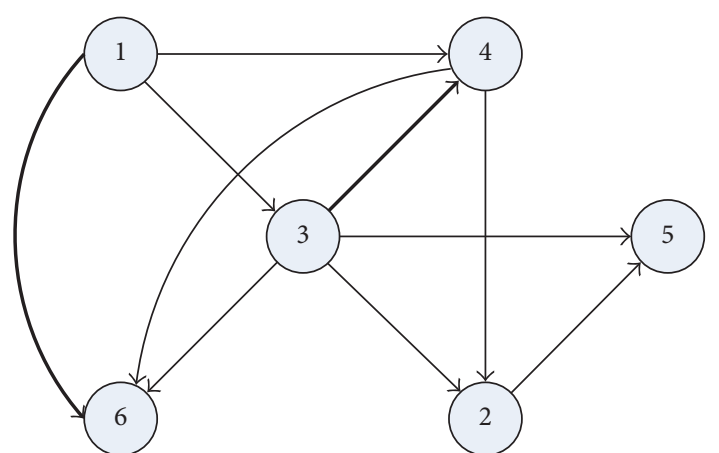

FIGURE 1: Drive network topology $(t<25)$.

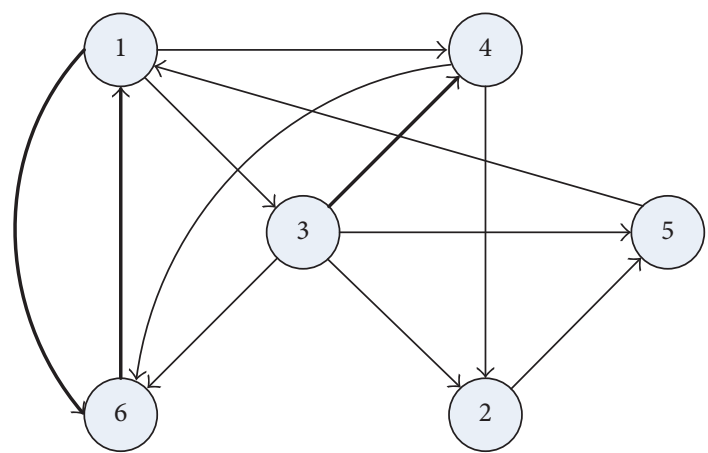

Figure 2: Drive network topology $(t>25)$.

A general complex dynamical network consisting of 6 identical nodes is chosen for the simulation. When $t<25$, the topological structure is described by Figure 1:

$$
C=\left(c_{i j}\right)_{6 \times 6}=\left[\begin{array}{cccccc}
0 & 0 & 0 & 0 & 0 & 0 \\
0 & -2 & 1 & 1 & 0 & 0 \\
1 & 0 & -1 & 0 & 0 & 0 \\
1 & 0 & 2 & -3 & 0 & 0 \\
0 & 1 & 1 & 0 & -2 & 0 \\
2 & 0 & 1 & 1 & 0 & -4
\end{array}\right] .
$$

We suppose that when $t>25$, the topology matrix of the drive system changes from Figures 1 and 2:

$$
C=\left(c_{i j}\right)_{6 \times 6}=\left[\begin{array}{cccccc}
-3 & 0 & 0 & 0 & 1 & 2 \\
0 & -2 & 1 & 1 & 0 & 0 \\
1 & 0 & -1 & 0 & 0 & 0 \\
1 & 0 & 2 & -3 & 0 & 0 \\
0 & 1 & 1 & 0 & -2 & 0 \\
2 & 0 & 1 & 1 & 0 & -4
\end{array}\right] \text {. }
$$

Applying the network with 6 identical nodes mentioned above to the network model (1), we get

$$
\begin{aligned}
& \dot{x}_{i}(t)=f\left(x_{i}(t)\right)+\sum_{j=1}^{N} c_{i j} L y_{j}(t-1), \\
& y_{i}(t)=H x_{i}(t),
\end{aligned}
$$

$$
i=1,2, \ldots, 6 \text {. }
$$

Treating the network model (17) as the drive system, we build the corresponding response system as follows:

$$
\begin{aligned}
\dot{\hat{x}}_{i}(t)= & f\left(\widehat{x}_{i}(t)\right)+\sum_{j=1}^{N} \widehat{c}_{i j} L \widehat{y}_{j}(t-1) \\
& -k_{i}\left(\hat{y}_{i}(t)-y_{i}(t-2)\right), \\
\hat{y}_{i}(t)= & H \widehat{x}_{i}(t),
\end{aligned}
$$

where $i=1,2, \ldots, 6, x_{i}(t)=\left[x_{i 1}(t), x_{i 2}(t), x_{i 3}(t)\right]^{T}$,

$$
\begin{aligned}
f\left(x_{i}(t)\right) & =\left[\begin{array}{c}
a\left(x_{i 2}(t)-x_{i 1}(t)\right) \\
c x_{i 1}(t)-x_{i 2}(t)-x_{i 1}(t) x_{i 3}(t) \\
x_{i 1}(t) x_{i 2}(t)-b x_{i 3}(t)
\end{array}\right], \\
\widehat{x}_{i}(t) & =\left[\widehat{x}_{i 1}(t), \widehat{x}_{i 2}(t), \widehat{x}_{i 3}(t)\right]^{T}, \\
L & =\left[l_{1}, l_{2}, l_{3}\right]^{T}, \\
H & =\left[h_{1}, h_{2}, h_{3}\right] .
\end{aligned}
$$

For simplicity, the coupling time delay is set as $\tau_{1}=1$, and the transmission time delay is set as $\tau_{2}=2$. Other parameters of the complex network are chosen as

$$
\begin{aligned}
& k_{i}=\left[\begin{array}{lll}
100 & 100 & 100
\end{array}\right]^{T}, \\
& L=\left[\begin{array}{lll}
1 & 1 & 1
\end{array}\right]^{T}, \\
& H=\left[\begin{array}{lll}
1 & 0 & 0
\end{array}\right] \text {. }
\end{aligned}
$$

The initial values of the drive and response system' state variables and all elements of the estimated topology matrix are set randomly in the interval $[0,1]$.

Since (A2) and (A3) hold, the response system (18) can detect the topology and track its changes of the drive system (17) with the control law (6). Meanwhile, the outputs of error dynamical system converge to zero as $t \rightarrow \infty$. Figure 3 shows the process of the response system detecting and tracking the target topology. The trajectories of outputs of the error dynamical system are shown in Figure 4.

From Figure 4, we can see that detecting the target topological structure and tracking its changes in real time are successful. The present research exposes the feasibility of achieving the topology identification in terms of inevitable coupling and transmission time delays when unexpected changes occur in the target network topology. 

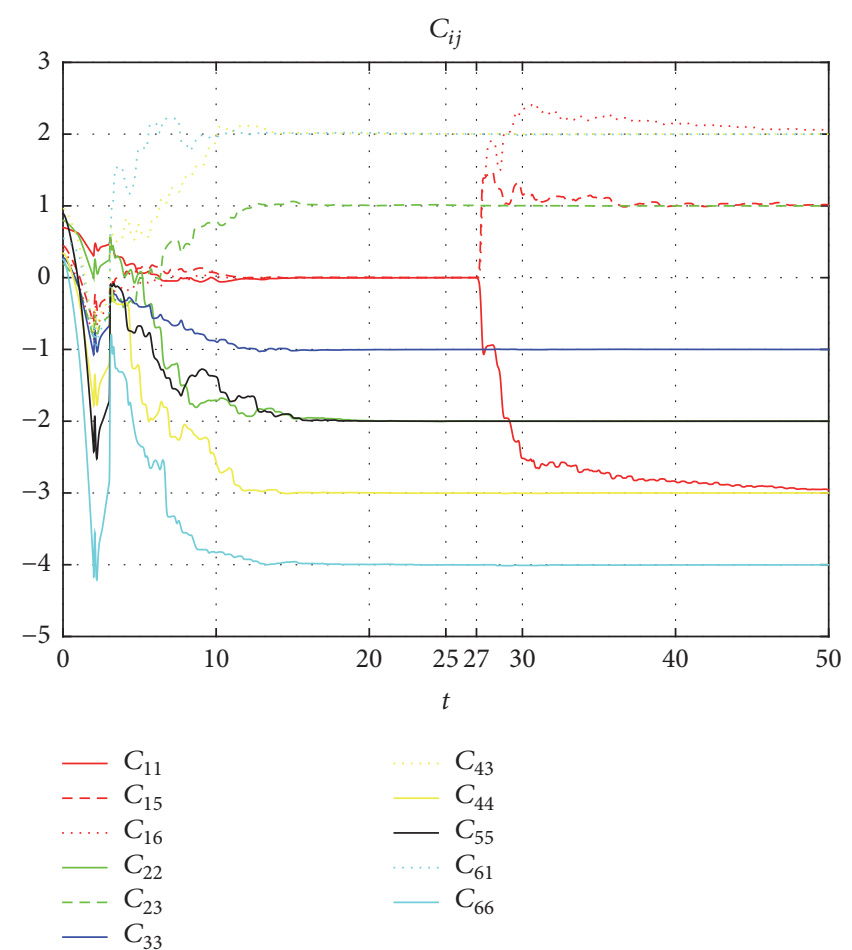

FIgURE 3: Detecting the topology of the drive system (17).
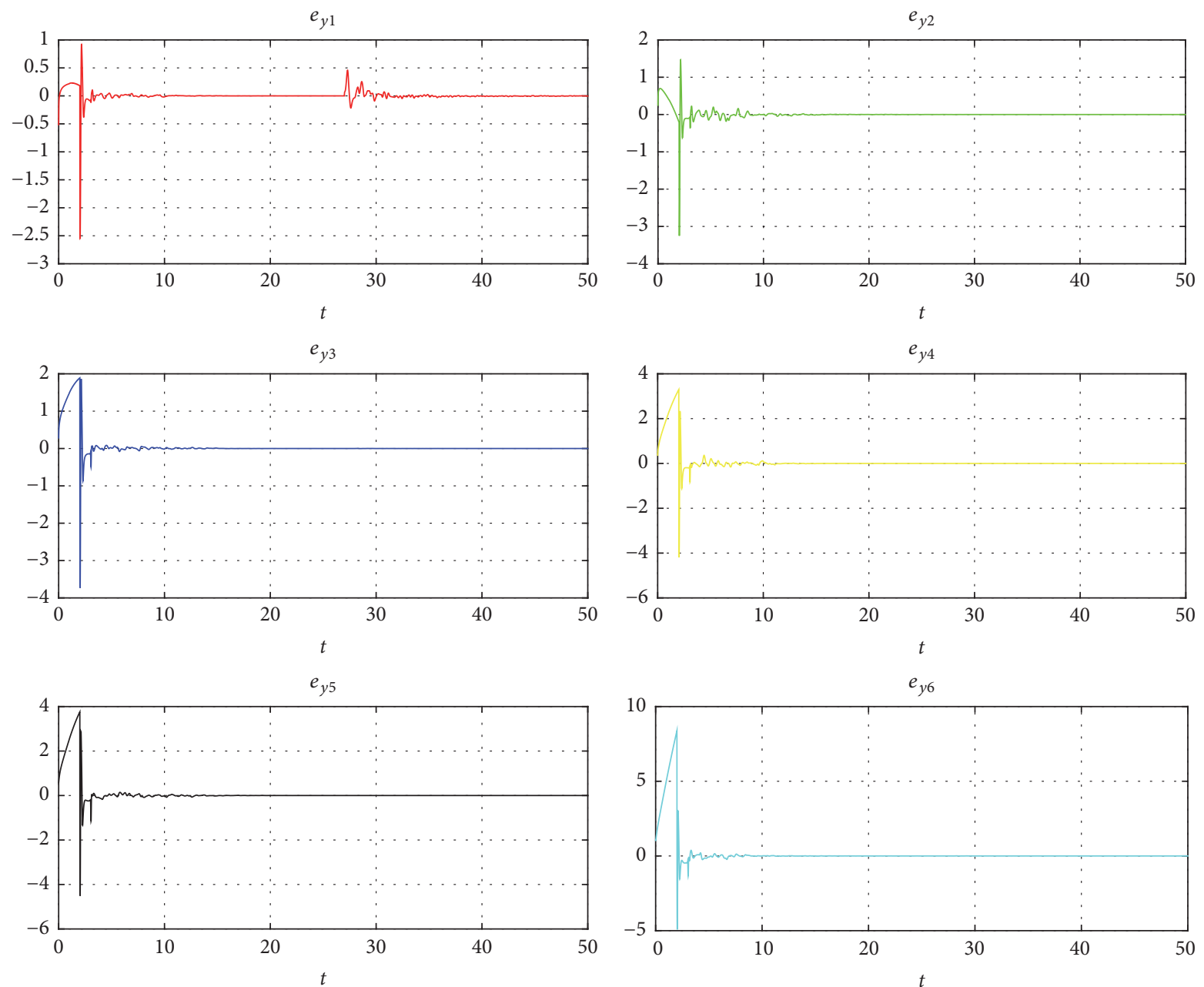

FIGURE 4: Trajectories of outputs of error dynamical system. The red line is the output $e_{y 1}$ of node $x_{1}$, the green line is the output $e_{y 2}$ of node $x_{2}$, the blue line is the output $e_{y 3}$ of node $x_{3}$, the yellow line is the output $e_{y 4}$ of node $x_{4}$, the black line is the output $e_{y 5}$ of node $x_{5}$, and the last line is the output $e_{y 6}$ of node $x_{6}$. 


\section{Conclusions}

In summary, a topology detection approach based on the state observer has been proposed in this paper. We transmit the outputs of the general complex dynamical network as the control signals. The coupling delay and transmission delay are both taken into account in the output-coupling complex network model at the same time. The transmission delay is not needed to be determined exactly, which is proved to have no influence on the topology-detected process. By means of LaSalle's invariance principle, we guarantee the asymptotic stability of the output error dynamical system and complete the derivation process. Numerical simulations have indicated that the target topology matrix can be completely estimated in terms of inevitable coupling and transmission time delays. Also, we can achieve the real-time monitoring of changes of the target network topology.

\section{Conflicts of Interest}

The authors declare that there are no conflicts of interest regarding the publication of this paper.

\section{Acknowledgments}

This work is supported by the National Natural Science Foundation of China (Grant nos. 61374180 and 61373136).

\section{References}

[1] S. H. Strogatz, "Exploring complex networks," Nature, vol. 410, no. 6825 , pp. 268-276, 2001.

[2] R. Cohen and S. Havlin, "Complex networks: structure, robustness and function. London, UK," Cambridge University Press, 2010.

[3] D. J. Watts, "The 'new' science of networks," Annual Review of Sociology, vol. 30, pp. 243-270, 2004.

[4] H. Jeong, B. Tombor, R. Albert, Z. N. Oltval, and A.-L. Barabásl, "The large-scale organization of metabolic networks," Nature, vol. 407, no. 6804, pp. 651-654, 2000.

[5] W. Yu, G. Wen, and X. Yu et al., "Bridging the gap between complex networks and smart grids," Journal of Control and Decision, vol. 1, no. 1, pp. 102-114, 2014.

[6] X. Li and G. Chen, "Synchronization and desynchronization of complex dynamical networks: an engineering viewpoint," IEEE Transactions on Circuits and Systems. I. Fundamental Theory and Applications, vol. 50, no. 11, pp. 1381-1390, 2003.

[7] D. J. Watts and S. H. Strogatz, "Collective dynamics of "smallworld” networks," Nature, vol. 393, no. 6684, pp. 440-442, 1998.

[8] A.-L. Barabási and R. Albert, "Emergence of scaling in random networks," Science, vol. 286, no. 5439, pp. 509-512, 1999.

[9] S. Boccaletti, V. Latora, Y. Moreno, M. Chavez, and D.-U. Hwang, "Complex networks: structure and dynamics," Physics Reports. A Review Section of Physics Letters, vol. 424, no. 4-5, pp. 175-308, 2006.

[10] M. E. Newman, "The structure and function of complex networks," SIAM Review, vol. 45, no. 2, pp. 167-256, 2003.

[11] R. Albert and A.-L. Barabási, "Statistical mechanics of complex networks," Reviews of Modern Physics, vol. 74, no. 1, pp. 47-97, 2002.
[12] A. Arenas, A. Díaz-Guilera, J. Kurths, Y. Moreno, and C. Zhou, "Synchronization in complex networks," Physics Reports, vol. 469, no. 3, pp. 93-153, 2008.

[13] J. Lü and G. Chen, "A time-varying complex dynamical network model and its controlled synchronization criteria," Institute of Electrical and Electronics Engineers. Transactions on Automatic Control, vol. 50, no. 6, pp. 841-846, 2005.

[14] G.-P. Jiang, W. K. Tang, and G. Chen, "A state-observer-based approach for synchronization in complex dynamical networks," IEEE Transactions on Circuits and Systems. I. Regular Papers, vol. 53, no. 12, pp. 2739-2745, 2006.

[15] J. Zhou, J.-a. Lu, and J. Lü, "Pinning adaptive synchronization of a general complex dynamical network," Automatica, vol. 44, no. 4, pp. 996-1003, 2008.

[16] C.-X. Fan, G.-P. Jiang, and F.-H. Jiang, "Synchronization between two complex dynamical networks using scalar signals under pinning control," IEEE Transactions on Circuits and Systems. I. Regular Papers, vol. 57, no. 11, pp. 2991-2998, 2010.

[17] C.-X. Fan and G.-P. Jiang, "State estimation of complex dynamical network under noisy transmission channel," in Proceedings of the 2012 IEEE International Symposium on Circuits and Systems, ISCAS 2012, pp. 2107-2110, kor, May 2012.

[18] C. Fan and G. Jiang, "State Reconstruction for Complex Dynamical Networks with Noises," International Journal of Modern Nonlinear Theory and Application, vol. 01, no. 01, pp. $1-5,2012$.

[19] L. Wang, G. Wei, and H. Shu, "State estimation for complex networks with randomly occurring coupling delays," Neurocomputing, vol. 122, pp. 513-520, 2013.

[20] J. Liang, Z. Wang, and X. Liu, "State estimation for coupled uncertain stochastic networks with missing measurements and time-varying delays: The discrete-time case," IEEE Transactions on Neural Networks, vol. 20, no. 5, pp. 781-793, 2009.

[21] J. Zhou and J.-a. Lu, "Topology identification of weighted complex dynamical networks," Physica A. Statistical Mechanics and its Applications, vol. 386, no. 1, pp. 481-491, 2007.

[22] X. Wu, C. Zhou, G. Chen, and J.-A. Lu, "Detecting the topologies of complex networks with stochastic perturbations," Chaos, vol. 21, no. 4, Article ID 043129, 2011.

[23] S. Zhang, X. Wu, J.-A. Lu, H. Feng, and J. Lu, "Recovering structures of complex dynamical networks based on generalized outer synchronization," IEEE Transactions on Circuits and Systems I: Regular Papers, vol. 61, no. 11, pp. 3216-3224, 2014.

[24] X. Wu, "Synchronization-based topology identification of weighted general complex dynamical networks with timevarying coupling delay," Physica A: Statistical Mechanics and its Applications, vol. 387, no. 4, pp. 997-1008, 2008.

[25] J. Zhao, Q. Li, J.-A. Lu, and Z.-P. Jiang, “Topology identification of complex dynamical networks," Chaos. An Interdisciplinary Journal of Nonlinear Science, vol. 20, no. 2, 023119, 7 pages, 2010.

[26] H. Liu, J. Lu, and J. Lü, “Topology identification of an uncertain general complex dynamical network," in Proceedings of the 2008 IEEE International Symposium on Circuits and Systems, ISCAS 2008, pp. 109-112, May 2008.

[27] H. Liu, J. Lu, and J. Lü, "Identification in general complex delayed dynamical networks," in Proceedings of the 27th Chinese Control Conference, CCC, pp. 419-423, chn, July 2008.

[28] Y. Che, R. X. Li, C. X. Han et al., "Adaptive lag synchronization based topology identification scheme of uncertain general complex dynamical networks," The European Physical Journal $B$, vol. 85 , no. 8 , pp. $1-8,2012$. 
[29] C.-X. Fan, Y.-H. Wan, and G.-P. Jiang, “Topology identification for a class of complex dynamical networks using output variables," Chinese Physics B, vol. 21, no. 2, Article ID 020510, 2012.

[30] H. K. Khalil and J. W. Grizzle, Nonlinear systems, vol. 3, Prentice hall, Upper Saddle River, USA, 2002.

[31] L. Chen, J.-A. Lu, and C. K. Tse, "Synchronization: An obstacle to identification of network topology," IEEE Transactions on Circuits and Systems II: Express Briefs, vol. 56, no. 4, pp. 310-314, 2009.

[32] H. Liu, J.-A. Lu, J. Lü, and D. J. Hill, "Structure identification of uncertain general complex dynamical networks with time delay," Automatica, vol. 45, no. 8, pp. 1799-1807, 2009.

[33] W. Yu, G. Chen, J. Cao, J. Lü, and U. Parlitz, "Parameter identification of dynamical systems from time series," Physical Review E - Statistical, Nonlinear, and Soft Matter Physics, vol. 75, no. 6, Article ID 067201, 2007. 


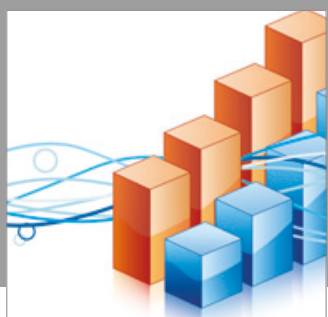

Advances in

Operations Research

vatersals

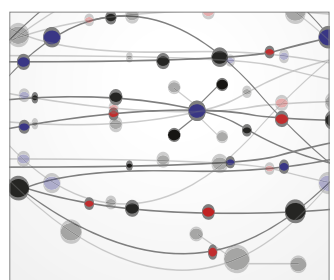

\section{The Scientific} World Journal
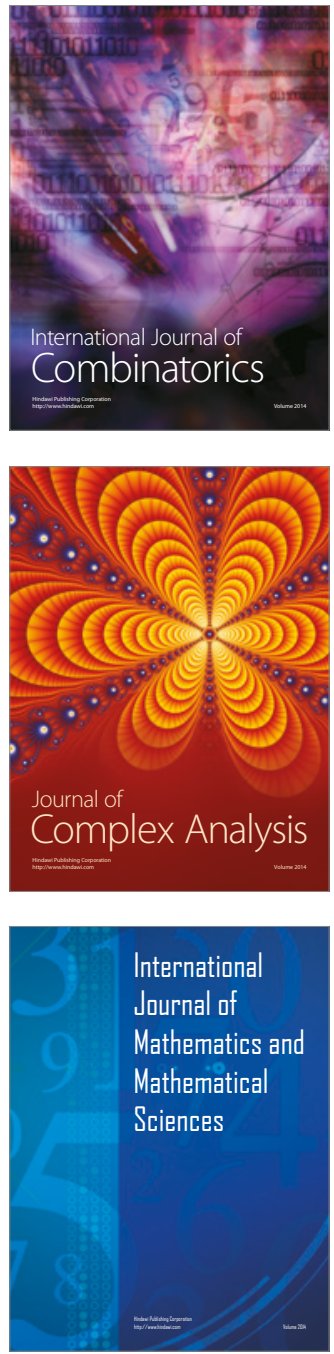
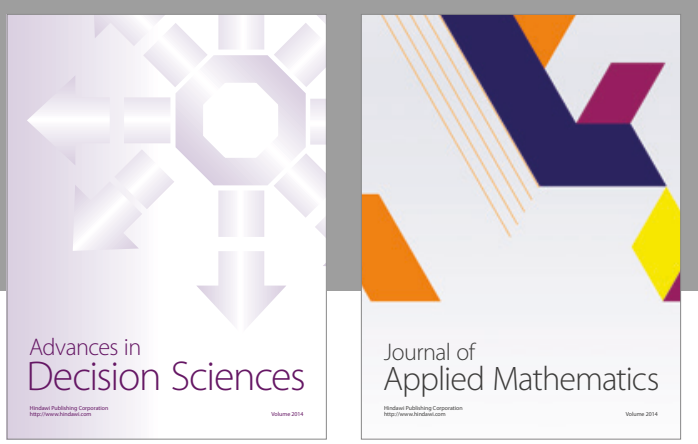

Algebra

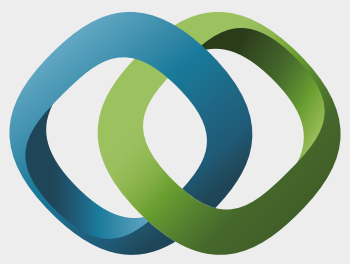

\section{Hindawi}

Submit your manuscripts at

https://www.hindawi.com
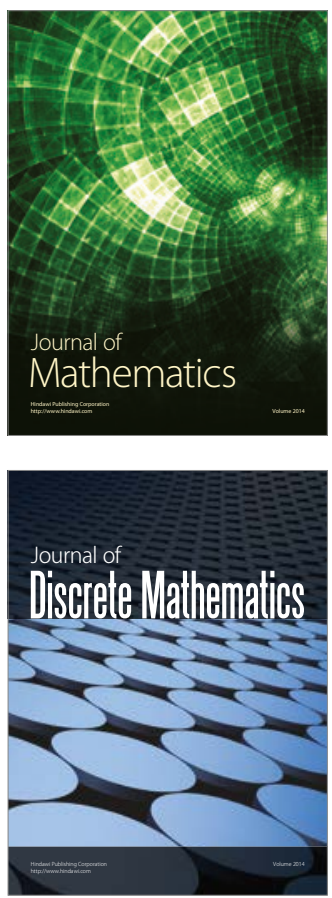

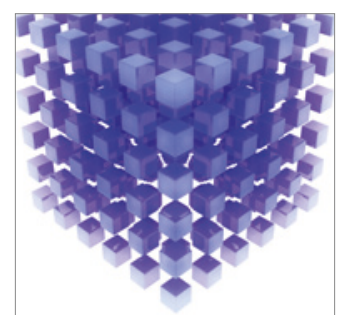

Mathematical Problems in Engineering
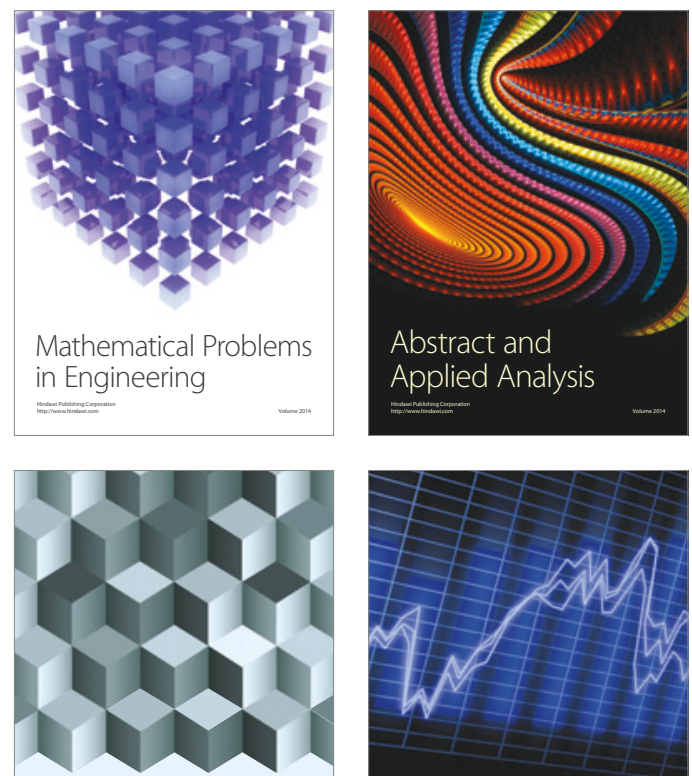

Journal of

Function Spaces

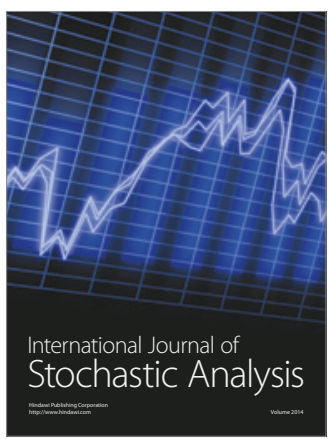

Probability and Statistics
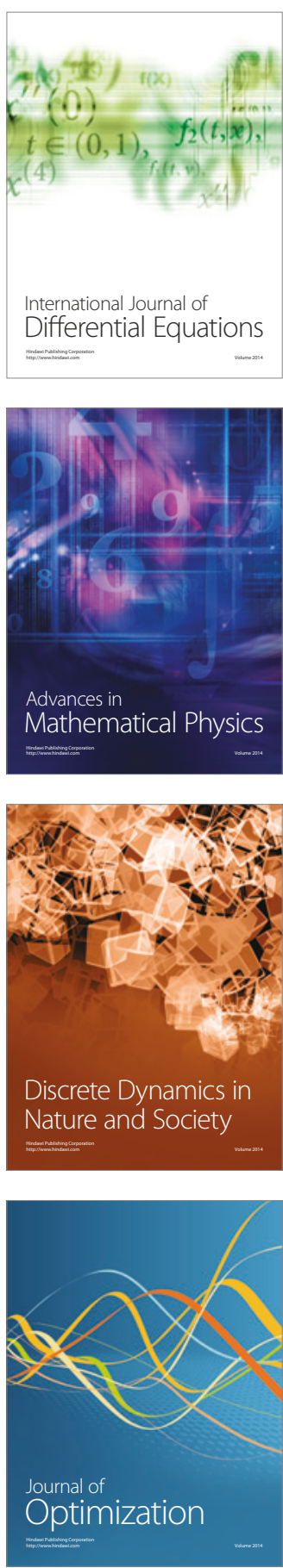